\title{
L'UTILISATION DES ANABOLISANTS DANS LA PRODUCTION DE VIANDE
}

\section{R. FERRANDO *}

\section{RESUME}

Il existe des cestrogènes dans l'herbe. Ils sont favorables à la croissance. Des anabolisants artificiels sont utilisés pour améliorer la prise de poids en embouche. Le diéthylstilboestrol, l'ostradiol associé à la testostérone ou à la progestérone et le trienbolone donnent des résultats positifís. Le bilan azoté est amélioré. La qualité des carcasses n'est pas diminuée, mais la viande paraît perdre plus de jus à la cuisson, surtout après emploi du D.E.S. Ce dernier produit a un pouvoir cancérogènc ainsi qu'une action sur le développement des organes géritaux mâles. La consommation de viande de veaux traités au D.E.S. entrâne chez les rats et souris une altération profonde de l'appareil génital.

Les associations à base d'cestradiol, le triembolone et le zéranol ne laissent persister dans les viandes que des résidus insignifiants. Mais les essais de "toxicité de relais " n'ont pas éte pratiqués.

L'emploi de tous les anabolisants dọt être réglementé.

\section{SUMMARY}

Use of anabolizing agents in meat production

Estrogens exist in grass. They are favorable to growth. Artificial anabolizing agents are used to improve weight gains in fattening. Diethylstilbostrol, oestradiol associated with testosterone or progesterone and trienbolone, give positive results. The nitrogen balance is improved. The quality of meal does not decrease, but the meat seems to losse more juice on cooking, especially after use of D.E.S. This last mentioned product has a carcinogenic (cancer-producing) power as well as an effect
on the development of the male genital organs. Consumption of meat of calves treated with D.E.S. induces in rats and mice a deep alteration of the genital organs.

Associations based on ostradiol, trienbolone or zeranol only leave insignificant residual traces in meat. But trials were not done on the "toxicity of relay".

The use of all anabolizers must be regulated by law.

Le développement démographique de certains continents et, parallèlement, l'élévation du niveau de vie d'autres pays, conjuguent leurs effets pour augmenter la demande en viande. On demeure inquiet en considérant qu'ici la viande est indispensable pour assurer l'équilibre protéique du régime de l'homme, alors que là, elle pourrait bientôt, cet équilibre étant obtenu, devenir une sorte de luxe. Il est bien évident que les conditions climatiques des pays gros consommateurs de viande les placent dans des conditions éminemment favorables à sa production. Tout concourt à améliorer cette production. Les conditions d'alimentation du bétail étant assurées, on peut rechercher quelles vont etre les substances capables d'accroître encore les rendements.

Il faut toutefois se persuader que les additifs ajoutés aux aliments des animaux d'élevage ne peuvent, en aucun cas, pallier les grands déficits de leurs rations bien que certains de ces additifs permettent d'économiser les protéines dans ces rations. On n'a pas assez insisté sur ce point. Peu d'expériences furent entreprises en vue de le mettre en évidence. On a surtout voulu accroître les rendements, élever la productivité, faire mieux que le voisin, sans chercher à concilier équilibre nutritif et équilibre écono-

(*) Laboratoire de Nutrition et d'Alimentation, E.N.V.A. mique. Il a fallu la récente crise du soja pour qu'on y songe en considérant enfin que de rares précurseurs ayant étudié cette question avaient eu raison d'attirer, depuis longtemps, l'attention sur cette conséquence de l'usage des additifs.

En Afrique, pour des raisons sur lesquelles il est inutile d'insister, cet aspect apparaît encore plus important qu'en Europe. Additifs et anabolisants doivent toujours être expérimentés puis employés en vue d'économiser les protides dans les rations animales en permettant, grâce à leurs propriétés phar. macologiques, d'en mettre davantage à la disposition de l'homme.

Nous voulions attirer l'attention sur ce point. Il nous semble d'une importance considérable tout en étant étroitement lié au sujet que nous allons maintenant aborder.

On considère comme anabolisant toute substance susceptible de favoriser la transformation des matières nutritives en tissus vivants. Plus spécialement, le pouvoir d'un anabolisant doit se caractériser par une accélération de la biosynthèse des protéines tissulaires avec, comme conséquence, le développement musculaire, la formation de la trame protidique de l'os et, parallèlement, une diminution du tissu adi- 
peux. Cette activité pourrail également déterminer, selon divers auteurs, une certaine hypertrophie et hyperplasie du système réticulo-endothélial ainsi qu'une baisse de l'élimination des électrolytes. Ces dernières propriétés ne sont pas unanimement admises.

Le type des anabolisants est la Testostérone, dont des modifications de la molécule changent ses diverses propriétés.

Il serait ainsi logique de qualifier anabolisant toute substance de nature hormonale ou non qui, chez les animaux, favorise l'anabolisme, c'est-à-dire, finalement, le développement. La nature hormonale du composé l'emporte toutefois dans la conception admise pour son utilisation. L'emploi, naguère, dans de nombreux pays et sur une large échelle, d'une substance cestrogène, comme le Diéthylstilboestrol (DES), produit de synthèse n'ayant rien à voir avec une hormone naturelle mais, pourtant le plus souvent aunsi dénommé, a contribué à augnenter ce malentendu. Dans le cas d'autres produits la confusion demeure aisée à faire par suite des actions secondaires et de ces substances, en particulier des actions cetrogènes.

On qualifie donc souvent, daus le langage courant, hormones, des composés employés à des fins d'ana- bolisme bien que ceux qui préparent des anabolisants essaient, au contraire, d'éviter toute allusion à unc action hormonale. Certes, en analysant les modalités d'actions au niveau des sites récepteurs, et dans une conception relevant de la biologie moléculaire, le terme d'hormone se justifie. Il s'agit ici de langage de spécialiste. Il s'est pourtant vulgarisé et, sur le plan du consommateur, donna lieu à une série de quiproquos à l'origine de la diffusion dans la presse d'énormités sensationnelles.

Nous venons de voir que le type même des anabolisants est bien une hormone, la Testostérone. Encore celle-ci possède, en même temps, un pouvoir virilisant. On a donc été conduit à rechercher divers composés dont l'action anabolisante dépasse l'action androgène ou, pour s'exprimer autrement, dont le rapport action anabolique/action androgène est favorable à la première influence, voire même exclusif de la seconde. Des modifications de la molécule permettent de parvenir plus ou moins à ce but (fig. 1). On agit ainsisi pour d'autres substances en s'efforçant de sélectionner celles n'ayant qu'un pouvoir anabolisant à l'exclusion de tout autre ou bien ne possédant, en même temps que cette action, aucune autre influence nuisible pour l'animal d'élevage, bien entendu, mais, surtout, à travers lui, pour le consommaLeur.

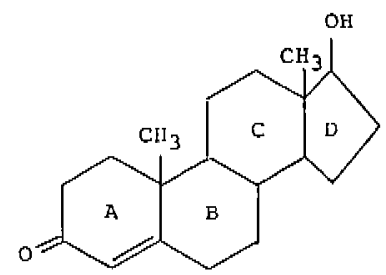

TESTOSTERONE

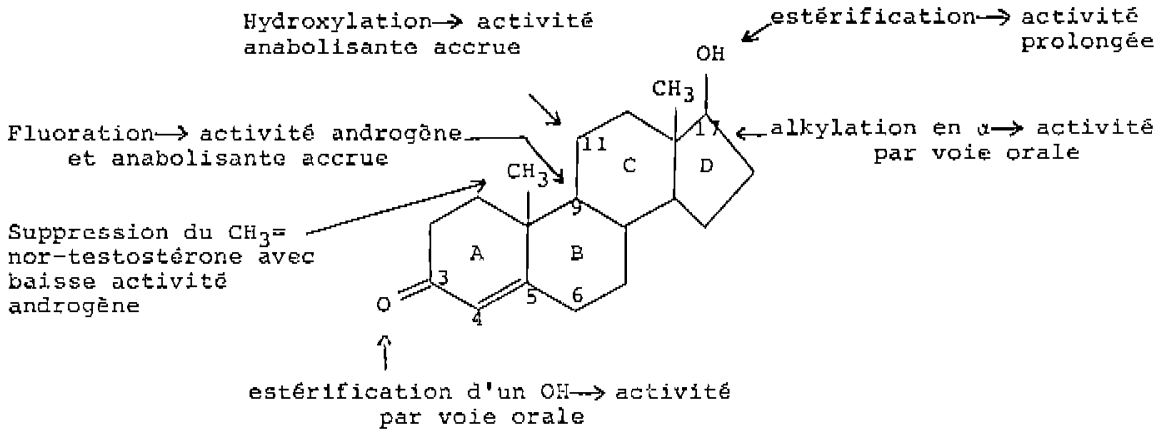

Figure 1

Modifications chimiques et modifications d'activité.

Peu importe d'ailleurs que, chez l'animal d'élevage, l'action anabolique recherchée d'abord s'accompagne d'autres actions secondaires n'influençant ni les performances, ni la qualité des viandes, ă condition que les produits, provenant de cet animal et destinés à la consommation de l'homme, soient exempts pour lui de toutes activités dangereuses à court, moyen et long termes.

Il convient, en effet, de bien souligner qu'un produit anabolisant, sans activités nuisibles surajoutées chez l'animal d'élevage, peut, à la suite des réactions métaboliques dont il est l'objet ou l'agent à travers l'organisme de l'animal, devenir néfaste pour le consonomateur. L'inverse est également vrai. Telle est d'ailleurs la base biologique de la méthodologie dite "Toxicité de relais" (FERRANDO et TRUHAUT).

Il est donc interdit d'utiliser en élevage un anabolisant n'ayant pas fait l'objet d'une expertise complète concernant l'ensemble de ses propriétés directes comme de ses éventuelles actions induites par relais. Telle est la mise au point que nous désirions faire au début de cet exposé dont le plan s'ordonnera ainsi :

I) Quelques anabolisants. Avantages de quelquesuns d'entre eux; leur étude comparée;

II) Les inconvénients de 1'usage des anabolisants;

III) Conclusions générales.

\section{I. - OUELOUES ANABOLISANTS. AVANTAGES DE QUELQUES-UNS D'ENTRE EUX ; LEUR ETUDE COMPAREE}

Un très large usage de substances à actions anabolisantes a été fait dans de nombreux pays mais 
notamment aux Etats-Unis. Citons, à ce sujet, les publications de BURROUGHS et coll. (1955); BEESON et coll. (1956); KARG (1962-1964) ; GOODRICH et coll (1967) ; RALSTON et coll. (1969); BAKER et ARTHAUD (1972) ; BRADLEY et coll. (1972), etc. Mentionnons également une publication de l'Académie Nationale des Sciences et du National Research Council en 1966. A moins de surcharger cet exposé, il est difficile d'énumérer toutes les études effectuées. Il est pourtant intéressant de signaler quelques travaux plus récents dus à POTTIE (1972), POKROVSKII et coll. (1972, PADUCHEVA (1972), GRANDADAM et coll. (1972-1973). Signalons enfin le compte rendu d'un symposium international sur ce problème, tenu, en 1972, à notre laboratoire.

Avant d'aborder l'examen des principaux anabolisants essayés expérimentalement ou employés en élevage, nous soulignerons que certains aliments naturels présentent des actions analogutes dues à des substances conférant en même temps à ces aliments un pouvoir oetrogène plus ou moins grand. La luzerne, les trèfles, le pâturiñ la sauge, mais, également, le palmiste, l'orge, l'avoine, le blé, pour ne citer que quelques plantes provoquant de telles actions, d'ailleurs variables, selon les conditions de cultures, comme nous l'avons mis nous-même en évidence en 1961 pour le blé et les carottes. Citons également le pouvoir estrogène de certaines moisissures du genre Fusarium qui ne semblent pas, pour autant, avoir une action anabolisante. $\mathrm{La}$ production d'oestrogène de ces moisissures ne paraît se produire qu'avec certains substrats de culture, millet, riz, orge, seigle, avoine, tournesol, selon CANTINI et coll. (1973) et, bien entendu, le maïs comme l'ont montré MIROCHA et coll. en 1967.

En 1945 GLEY et MENTZER avaient d'ailleurs préparé des coumarines otrogènes. Les substances actives présentes à l'état naturel dans les plantes sont en général des composés phénoliques appartenant à différents groupes.

Dans celui des isoflavones, on trouve la Génistéine et des dérivés comme le Prunetol, la Biochanine A, qui serait transformée en Génistéine par les bactéries du rumen, la Prumetine, la Daidzeine et la Formononétine. Toutes ont une activité œstrogène.

Dans le groupe des coumarines, nous citerons le Coumestrol, qui serait 30 à 40 fois plus actif que la Génistéine, et qui a été isolé du trèfle et de la luzerne. Citons aussi le 3 - ( $\mathrm{p}$ - hydroxyphényl) - 4 propyl 7 - hydroxy - coumarine synthétisé par MENTZER mais existant également à l'état naturel.

A propos de la luzerne, et en relation avec la présence de ces substances, MATSUSHIMA et CLAUTON (1960) notent qu'en la distribuant à des bcufs à l'engrais, après séchage artificiel, à des taux de 1,25 2,5 et 5 livres anglaises par tête et par jour, on augmente les performances de ces animaux parallèle- ment à l'élévation du taux de luzerne dans la ration. L'efficacité du DES ajouté en supplément à ces rations s'abaisse au fur et à mesure que les quantités de Juzerne distribuées augmentent. La luzerne séchée artificiellement et celle séchée au soleil ont la même action favorable sur la prise de poids.

Ces composés phénoliques naturels se détoxiquent dans l'organisme grâce à divers processus :

Glycuroconjugaison,

Sulfoconjugaison,

0 - méthylation dans quelgues cas spéciaux,

Hydroxylation de la chaîne aromatique.

Dans le cas des hydroxycoumarines, 8 à 28 p. 100 seraient excrétées sous forme de sulfoconjugués et 50 à 80 p. 100 sous forme de glycuroconjugués. Il existe une circulation entérohépatique des glycuroconjugués. En effet, une partie de ceux-ci étant hydrolysée par les glycuronidases des bactéries du tube digestif se trouve réabsorbée, l'autre partie étant rejetée avec les fèces.

Malgré leur action anabolisante, ces composés ont provoqué des accidents comme ceux d'infécondité observés en Australie avec le trèfle souterrain et classiquement connus. Plus récemment, STANDARA et CHURY (1973) ont mis en évidence du Coumestrol dans le sperme de lapins consommant de la luzerne. Ce Coumestrol serait responsable de la dégénérescence de l'ouf et de désordres de la fécondation observés, chez les lapines, dès après la sailliẹ.

Certaines substances naturellement présentes dans les plantes appartiennent au groupe des stérols. Parmi elles, on trouve l'Gstrone, isolée en 1933 par BUTENAND et JACOBI de la noix de palme et l'Estriol qui fut extrait des chatons de saules.

Nous avons tenu à souligner l'cxistence, dans les plantes, de produits à action ostrogènc susceptibles d'être néfastes mais ayant aussi parfois, comme dans le cas de la luzerne, des propriétés anabolisantes. La diffusion dans la nature de ces composés est remarquable. Enfin, n'ayons garde d'oublier que quelques vitamines, comme les vitamines $A, E, B_{6}$ et $B_{12}$ présentent une certaine action anabolisante sinon une action anabolisante certaine, associée à un pouvoir cestrogène (SHARAF et GOMAA, 1971).

Les anabolisants le plus couramment utilisés en élevage sont les suivants :

- Le Diéthylstilboestrol (DES), associé parfois à la Testostérone ;

- L'association d'hormones naturelles produites par synthèse: Estradiol, Progestérone ou EstradiolTestostérone:

- L'association benzoate d'Estradiol-Trienbolone;

- Le Zéranol ou Lactone du 6 - 6,10 - dihydroxyundecyl) de l'acide $\beta$ résorcylique (fig. 2 et 3 ).

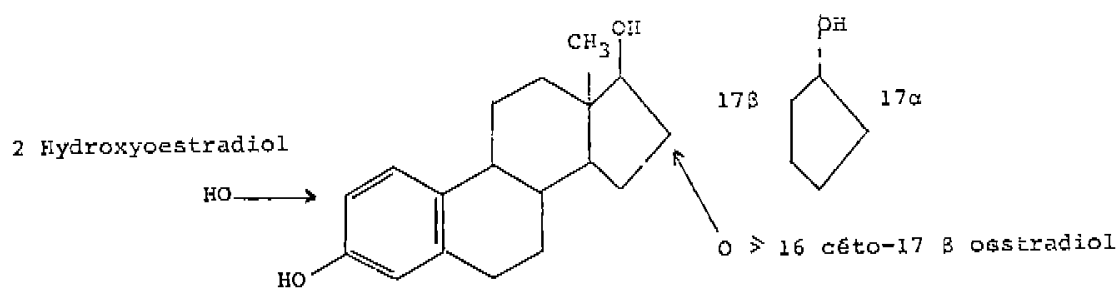

OESTRADIOL et gूos DERIVES 


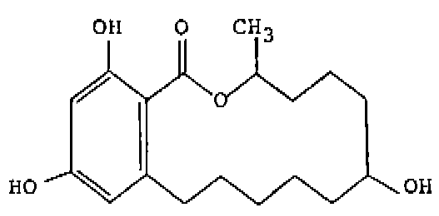

4-lactone du 6-(6,10-dihydroxy-undecyl)-8-resorcylique (ZERANOL)<smiles>CC(=O)OC1CCC2C3CCC4=CC(=O)CCC4=C3C=CC12C</smiles>

ACETATE DE TRENBOLONE

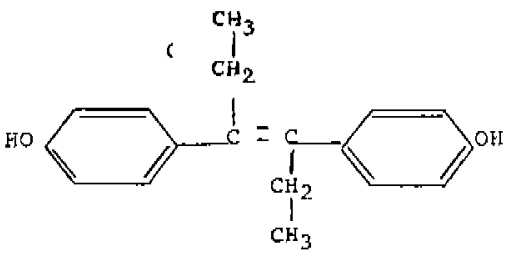

DIETHYLSTTLBOESIROL (DES)

Figure 3

Anabolisants de synthèse.

En dehors de yuelques résultats enregistrés aux Etats-Unis, nous parlerons surtout de ceux obtenus en Europe de l'Ouest, selon les conditions d'élevage qui $\mathrm{y}$ sont pratiquées.
Les importantes expériences effectuées par VAN DER WAL concernent un mal de 563 veaux mâles de race Frisonne. Ces animaux avaient été achetés à l'âge de cinq jours après avơir reçu le colostrum. Ils ont permis de comparer l'effet de diverses substances, à savoir :

- Le DES, injecté à raison de $25 \mathrm{mg}$ ou de $100 \mathrm{mg} /$ sujet à l'âge de 9 semaines.

D'autres groupes recevant les mêmes doses à 9 semaines, puis, une nouvelle dose, à l'âge de 13 semaines:

- L'Estractiol-Testostérone (20 mg + $200 \mathrm{mg})$ ou l'Estradiol-Progestérone $(20 \mathrm{mg}+200 \mathrm{mg})$ adnuinistrés à l'âge de 11 semaines et comparés avec le DES utilisé à raison de $24 \mathrm{mg}$ au même âge ;

- Le Zéranol et l'acétate de Trenbolone aux doses respectives de $36 \mathrm{mg}$ et $40 \mathrm{mg}$ entre 11 et $17-18$ semaines. Ces deux produits furent comparés au mélange Estradiol-Testostêrone ;

- L'Estradiol-Trenbolone (20 $\mathrm{mg}+140 \mathrm{mg}$ ) en comparaison avec l'Estradio-Testostérone (20 $\mathrm{mg}+$ $200 \mathrm{mg})$, l'Estradiol-Progestérone (20 $\mathrm{mg}+200 \mathrm{mg})$, l'Estradiol seul (20 mg) entre 11 et 17-18 semaines.

Des témoins non traités existaient dans tous les lots.

Tous les animaux qui recevaient un lait de remplacement furent abattus entre 150 et 180 kilos vifs.

Les effets sur la croissance sont peu sensibles quand or utilise une seule dose de DES de $25 \mathrm{mg}$ ou 2 fois $100 \mathrm{mg}$. Le moment de l'administration a cependant une grande importance sur l'effet obtenu. Le DES, utilisé chez des sujets âgés de 5 semaines, améliore la croissance par rapport aux témoins mais entraîne par la suite son ralentissement. Finalement, les témoins pèsent souvent plus que les sujets traités aty DES. Une implantation plus tardive, faite à l'âge de 9 semaines, améliore au contraire nettement la croissance. Il existe cependant des écarts en fonction de la durée du traitement comme le montre le tableau 1.

Gain de poids de veaux implantés au DES au cours de la période de temps variable (selon Van Der Wal et collab.)

\begin{tabular}{|c|c|c|c|c|}
\hline \multirow{2}{*}{$\begin{array}{l}\text { Onsa de DES (mg) } \\
\text { et đ̃ge d'implentation }\end{array}$} & \multicolumn{2}{|c|}{$\begin{array}{l}\text { Période de } \\
9 \text { à } 13 \text { semaines } \\
\text { [4 semaines }\end{array}$} & \multicolumn{2}{|c|}{$\begin{array}{l}\text { Pertode da } \\
\text { g } \mathrm{g} 15 \text { semaines } 1 / 2 \\
\text { [6,5 semaines }]\end{array}$} \\
\hline & ks & $\begin{array}{c}\text { P.100 } \\
\text { des témoins }\end{array}$ & $\mathrm{kE}$ & $\begin{array}{c}p .100 \\
\text { des têmoins }\end{array}$ \\
\hline têmains & 33,8 & 100 & 61,8 & 100 \\
\hline 25 ả 9 semeines & $39,3 *$ & 116,3 & $69,7 *$ & 171.8 \\
\hline 25 à 9 et à 13 semaines & 38,9 & 115,1 & $68,8 \ldots$ & 111,3 \\
\hline 100 à 9 semaines & $40,5 *$ & 119,8 & $69.6 *$ & $112, \mathrm{E}$ \\
\hline 100 à 9 et à 13 semaines & $40,7 *$ & 120,4 & $71,8 *$ & $11 \mathrm{~B}, 2$ \\
\hline
\end{tabular}

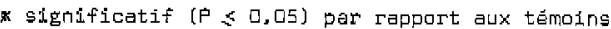

On notera qu'une seconde implantation faite à 13 semaines n'a guère d'influence sur le poids final à 15,5 semaines. Dans cette expérience l'indice de consommation fut, par rapport aux témoins, également amélioré de 5 à 7 p. 100 chez tous les animaux traités.

Quand les anabolisants sont administrés à l'âge de 11 semaines, ou même plus tardivement, VAN DER WAL obtient les améliorations de croissance suivantes par rapport aux animaux témoins.

Avec $25 \mathrm{mg}$ DES, après 4 à 5 semaines : 6,4 - 9,1 et 4,9 kilos par veau.

Avec $20 \mathrm{mg}$ Estradiol $+200 \mathrm{mg}$ Testostérone, après 3 à 4 semaines : 9,7 - 7,6 - 7,8 et 7,9 kilos par veau.
Avec $20 \mathrm{mg}$ Estradiol $+200 \mathrm{mg}$ Progestérone, après 4 à 5 semaines : 7,6 - 4,6 et 5,4 kilos par veau.

Avec $20 \mathrm{mg}$ Estradiol $+140 \mathrm{mg}$ Trienbolone, après 4 semaines : $10,5-15,8-12,6-10,4$ et 9,0 kilos par veau.

Avec $20 \mathrm{mg}$ Estradiol, après 2 à 4 semaines : 4,1 et 4,1 kilos par veau.

Avec $36 \mathrm{mg}$ de Zéranol, après 6 semaines : 0,5 et 3,4 kilos par veau.

Avec $40 \mathrm{mg}$ de Trienbolone on obtient, en fin d'expérience, des gains inférieurs à ceux des témoins.

VAN DER WAL signale que les essais américains et 
allemands, portant sur le Zéranol, aboutissent à de meilleurs résultats.

Nous pouvons, en effet, citer les chiffres de WILLWOCK (1972) qui, utilisant chez le veau des implants de $36 \mathrm{mg}$ de Zéranol, élève en 65 jours le poids vif de ces animaux de 8,1 p. 100 par rapport aux témoins. L'implantation avec le mélange Estradiol-Progestérone n'étant alors que de 1,7 p. 100 . Pourtant BORGER et coll. (1973) n'observent, après 169 jours d'implantation de bouvillons, qu'une différence de $90 \mathrm{~g}$ en faveur du Zéranol, quel que soit le taux protéique du régime distribué : 9,5 - 11,0 et 12,5 p. 100 .

Nous-mêmes, comparant, au cours de deux essais, le DAS aux mélanges Estradiol-Progestérone ou EEstradiol-Testostérone, sur un total de 142 veaux máles ou femelles répartis en 5 lots et implantés au moment de la mise en batterie puis, à nouveau, 60 jours plus tard pour être abattus après un délai de 38 jours, soit presque 5,5 semaines, avons obtenu les résultats rassemblés dans les tableaux 2 et 3 .

2

Nature des implants utilisés chez les veaux et résultats zootechniques obtenus au cours du premier essai

\begin{tabular}{|c|c|c|c|c|c|}
\hline $\mathrm{N}^{Q}$ des lots & 1 & 2 & 3 & $\therefore$ & 5 \\
\hline Nambre et sexe & 13 màles & 13 mäles & 15 femelles & 13 māless & 15 femellas \\
\hline Implants (mg) & $\left|\begin{array}{c}\text { D.E.S. } \\
\text { (24 } \\
m . E \times 2\end{array}\right|$ & $\mid \begin{array}{l}\text { Progestérone } \\
\text { Oestzadiol } \\
(200-20) 2 \\
\text { 䗆 } \mathrm{mg}\end{array}$ & $\mid \begin{array}{c}\text { Testontemone } \\
\left.0 a 5 t r d d^{2} 0\right] \\
(200+20) 2 \\
m g \text {-g }\end{array}$ & $\begin{array}{c}\text { têmoins } \\
\square\end{array}$ & $\begin{array}{c}\text { témains } \\
\square\end{array}$ \\
\hline $\begin{array}{l}\text { Paids moyens [kg] } \\
\text { au debut de I'essai }\end{array}$ & 44,6 & 44,7 & 45,2 & 44,7 & 45.5 \\
\hline $\begin{array}{l}\text { Paids moyens }(k \mathrm{k}) \\
\text { après } 98 \text { jours }\end{array}$ & 102,3 & 142,9 & 143.2 & 136,2 & 138,1 \\
\hline $\begin{array}{l}\text { Poids moyens }[\mathrm{kg} \text { ] } \\
\text { des careasses } *\end{array}$ & a7,3 & 88,1 & 87,6 & 84,3 & 32,5 \\
\hline $\begin{array}{l}\text { Gain de poids } \\
\text { en carcasse (kg) }\end{array}$ & 3,0 & 3,8 & 5,1 & - & - \\
\hline Rendement p.100 & 61,2 & $E 1,6$ & 61,2 & 60.9 & 59,7 \\
\hline $\begin{array}{l}\text { Note moyentie } \\
\text { couleur-confarmation } \\
\text { engralssement }\end{array}$ & 7,99 & 7.75 & 7.45 & 7.03 & 7,32 \\
\hline
\end{tabular}

3

Nature des implants utilisés chez les veaux et résultats zootechniques obtenus au cours du second essai

\begin{tabular}{|c|c|c|c|c|c|}
\hline$N^{0}$ des lots & 1 & 2 & 3 & 4 & 5 \\
\hline Nambre et sexe & 15 males & $13 \mathrm{māles}$ & 16 femelles & 14 māles & 15 femelles \\
\hline Implents (m) & $\mid \begin{array}{c}\text { D.E.5. } \\
{[24 \text { mg } \times 2]}\end{array}$ & $\mid \begin{array}{c}\text { Frogesterone } \\
\text { Oestradiol } \\
(200+20) 2 \\
\mathrm{mg} \mathrm{mg}\end{array}$ & $\begin{array}{c}\text { Testagtérone } \\
\text { 0estracilol } \\
{[200+20] 2} \\
\mathrm{mg} \mathrm{mg}\end{array}$ & témains & $\begin{array}{c}\text { témoins } \\
0\end{array}$ \\
\hline $\begin{array}{l}\text { Polds moyens ( } \mathrm{kg} \text { ) } \\
\text { au dêtut de l'essai }\end{array}$ & 54,6 & 54,7 & 46,1 & 54,4 & 46,2 \\
\hline $\begin{array}{l}\text { Paids moyens }\{\mathrm{kg}\} \\
\text { apres } 95 \text { jours }\end{array}$ & 748,3 & $75 x, 3$ & 130,8 & 142,2 & 724,2 \\
\hline $\begin{array}{l}\text { Paids mayens }\{\mathrm{kg}\} \\
\text { des carcasses }\end{array}$ & $9 t, i$ & 53,2 & $7 \varepsilon, 6$ & 87,0 & 75,0 \\
\hline $\begin{array}{l}\text { Gain de poids } \\
\text { en darcasse ( } \mathrm{kg} \text { ) }\end{array}$ & 2,1 & 3,6 & 3,6 & - & - \\
\hline Rendement p. 100 & 51,7 & 51.5 & 59,5 & 67.2 & 60,4 \\
\hline $\begin{array}{l}\text { Note moyenne, } \\
\text { ouleur-canformation } \\
\text { engraissement }\end{array}$ & xx & xx & 7,31 & * $\mathbf{z}$ & N* \\
\hline $\begin{array}{l}\text { * différences 5ìgni } \\
\text { selan le sexe. }\end{array}$ & ative: & $r \cong 20 \pi$ & & ts ${ }_{n}^{n}$ & implantés" \\
\hline * le découpe fatte & les & nāl & Iats 1,2 & 4, pius & importanะe \\
\hline
\end{tabular}

Les essais effectués en Hollande montrent que l'association Estradiol-Trienbolone apparaît comme la plus efficace. Ceux effectués en France font apparaître une meilleure influence de l'association des hormones naturelles : Estradiol-Progestérone et Estradiol-Testostérone, comme ce fut le cas en Hollande, enfin celle du DES. En ce qui le concerne, la forme trans donne, selon BRADLEY et coll. (1972), de meilleurs résultats que la forme ci:

L'Estradiol employé seul est un peu moins effi- cace qu'en mélange avec la Progestérone ou la Testostérone.

Citons cles améliorations de gain de poids de $13,5 \mathrm{p}$. 100 et 17,4 p. 100 chez des veaux castrés, traités avec, respectivement, $48 \mathrm{mg}$ de DES et $36 \mathrm{mg}$ de DES + $50 \mathrm{mg}$ de Dianabol par GAVRISHCHUK et KALININA (1973). Enfin BOGDANOFF et KASNEDELTS. CHEFF (1972), utilisant l'acétate de Triénolone (17 $\beta$ acétoxy $\Delta 4,9,10,11,12$ Estradiène) à raison de $250 \mathrm{mg}$ en injection sous-cutanée, observent des gains de 
poids supérieurs de $150 \mathrm{~g}$ chez les mâles et de $50 \mathrm{~g}$ chez les femelles par rapport aux témoins. L'action dure 5 semaines. Le traitement doit intervenir 30 à 35 jours avant l'abattage. Finalement ces résultats sont peu encourageants *.

Les annéliorations de la croissance et de l'indice de consommation observées ont pour origine des changements du métabolisme. Des études ont été poursuivies sur cette question. La plupart concernent le DES dont on sait, depuis assez longtemps, qu'il entraîn des modifications du bilan azoté et de certains constituants biochimiques du sang. GREBING et coll. (1970) ont observé, sur 16 agneaux hybrides, que le DES abaissait l'excrétion azotée urinaire. Elle passe, dans les 3 et 5 jours suivant l'administration du produit, d'une moyenue quotidienne de $8 \mathrm{~g}$ à celle de $5 \mathrm{~g}$.

HUBER (1970) constate, toujours sur des agneaux, la même influence du DES mais, note également une baisse de l'urémie et des variations dans l'activité de l'arginase hépatique. DAVIS et GARRIGUS (1971) constatent les mêmes effets favorables sur le bilan azoté et l'urémie des agneatux.

Chez les bouvillons OLTJEN et coll. (1972) signalent également une meilleure rétention azotée chez les sujets traités au DES. Les résultats les plus probants sont obtenus avec les lots recevant 18,4 p. 100 d'équivalent protéique apporté par une ration combi. nant soja et urée.

Plus récemment, BRUEGGEMAN et coll. (1973), utilisaut d'autres anabolisants, ont mis en évidence que, chez le veau, $25 \mathrm{mg}$ de benzoate d'CEstradiol associés à $140 \mathrm{mg}$ de Trienbolone amélioraient la rétention azotéc.

TIEWS et coll. (1973) obtiennent, sur des veaux traités ou non traités par le même mélange d'Estradiol et de Trienbolone utilisé aux mêmes doses, des bilans azotés indiqués dans le tableau 4 .

\section{4}

Bilan azoté de veaux traités ou non par le moélange benzoate d'Estradiol $(25 \mathrm{mg})+$ Trienbolone $(140 \mathrm{mg})$ après 10 jours d'implantation et sur une durée de 5 jours

\begin{tabular}{|c|c|c|}
\hline & Tëmoin & sujet traité \\
\hline Poids du corps (kg) début & 134 & 143 \\
\hline Eacn par jour (g) & 1.500 & 2.600 \\
\hline N ingers $(g / j)$ & 109.9 & 115.8 \\
\hline N excrété feaces [g/j] & 5,7 & 5,4 \\
\hline N excrété urine $[g / j]$ & 51,7 & 35.5 \\
\hline$N$ retenu $[g / j]$ & 52,5 & 74,9 \\
\hline g de $N$ retenu/kg gain vif & 35 & 28,8 \\
\hline
\end{tabular}

Les chiffres de ce tableau confirment les observations faites par BRUGGEMANN travaillant en collaboration avec TIEWS qui sotilignent, qu'au-delà d'un certain gain de poids quotidien, la quantité d'azote retenue par kilo de gain tend à s'abaisser.

TIEWS observe d'ailleurs que, chez des animaux non traités des phénomènes analogues pourraient être observés. Dans ses études de bilans, VAN DER WAL (1973) souligne que, dans la plupart des cas, la meilleure rétention azotée se produit 4 à 5 semaines après l'implantation. Il existe alors une bonne corrélation entre l'efficacité des anabolisants sur la croissance, l'indice de consomination et la rétention azotée. Les moyennes des rétentions azotées sont les suivantes :

— Estradiol + Trienbolone : $(25+140) 546$ g après 38 jours:

- Estradiol + Testostérone : $(20+200) 139$ et $239 \mathrm{~g}$ après 28 et 24 jours respectivement;

- DES (120 mog) : 212 et $208 \mathrm{~g}$ après 29 jours dans les deux cas;

- Estradiol (20 mg) : 187 et $159 \mathrm{~g}$ après 38 et 24 jours respectivement.

Le Zéranol, la Testostérone, la Progestérone et la Trienbolone, utilisées isolément, ne modifient pas la rétention azotée.

L'ensemble de ces résultats met en évidence l'amélioration de l'anabolisme protéique à la suite de l'usage de quelques-uns de ces composés. Il confirme d'autres observations faites antérieurement sur les animaux des espèces bovine et ovine.

L'action des anabolisants ne se bornerait pas setlement à une augmentation de la prise de poids. Elle

(*) Un trouvera dans le compte rendu de ce colloque d'intéressants résultats obtenus par SERRES, à Madagascar, sur les animaux âgés de 7 ans $1 / 2$ et traités à l'acétate de trienbolone. pourrait se renforcer d'une amélioration des détenses de l'organisme. C'est ainsi que $1 \mathrm{mg}$ de Stitboestrol en injection sous-cutanée stimule l'activité phagocytaire du foie de la souris à l'égard des érythrocytes de moutons tout en l'abaissant dans la rate de ce même animal. Finalement, d'après WARR et SLJIVIC (1973), les phénomènes d'immunosuppressions tendraient à l'emporter. LOOSE et coll. (1973) ont également observé que, chez le poulet, l'injection intramusculaire de $500 \mu \mathrm{g}$ de DES diminue significativement, en modifiant la réactivité du système réticulo-endothélial, la phagocytose provoquée par l'infection due à Plasmodizm lophurae. La gravité de la maladie s'en trouve accrue.

Citons enfin LAZARD (1973) qui montre, chez le rat traité au DES ou à l'acétate d'Gstradiol, l'absence de stimulation de l'activité phagocytaire du système réticulo-endothélial. Chez la souris l'Estradiol élèverait pourtant cette activité.

On ne peut qu'enregistrer des différences interspécifiques, et il n'est guère possible, dans l'état actuel de nos informations, de porter un renforcement des défenses de l'organisme à l'actif des anabolisants.

Les avantages, variables suivant les produits utilisés, demeurent pourtant évidents quand on considère le gain de poids et l'amélioration de l'anabolisme protéique. Pour y parvenir, il est tout de même essentiel de se placer dans certaines conditions et, en particulier, de choisir l'époque de l'administration du produit, pour obtenir les meilleurs effets. On regrettera cependant qu'aucun chercheur n'ait tenté d'exploiter les propriétés des anabolisants, non pour accroître les performances, mais pour réduire le taux des protéines des régimes en obtenant tout de même des rendements favorables. L'économie ainsi réalisée sur le coût des rations compenserait sans doute largement le prix des anabolisants dont l'effet devrait 
alors se faire sentir au maximum. Nous avons pu effectuer de telles observations en utilisant, à faibles doses, les antibiotiques et d'autres facteurs de croissance. C'est là une voie de recherche intéressante à poursuivre dans tous les pays, en Afrique en particulier. Le monde manque de protéines. Il importe de les économiser au maximum.

A côté des avantages retirés de l'usage des anabolisants, il existe cependant quelques inconvénients. Certains d'entre eux ne sont pas graves. IIs ne compromettent pas l'utilisation pratique de ces adjuvants de l'élevage. D'autres apparaissent, au contraire, comme susceptibles de faire courir des risques aux consommateurs. Nous allons les examiner dans la seconde partie de cet exposé. Ceci nous permettra d'ailleurs d'envisager ensuite rapidement les modalités d'utilisation pratique de ces produits et les aspects légaux de leur emploi.

\section{II. - LES INCONVENIENTS}

\section{DE L'USAGE DES ANABOLISANTS}

La qualité des productions provenant des animaux traités avec des anabolisants a donné, donne et donnera encore lieu à quelques polémiques. On doit tout d'abord mentionner que, selon PRESTON (1969), chez des agneaux implantés au DES, l'eau corporelle totale n'est pas modifiée. Son renouvellement serait même, par rapport aux témoins, de 29 p. 100 inférieur chez les sujets traités. L'azotémie est également réduite, comme l'ont constaté d'autres auteurs (cf. plus haut).
Qu'en est-il de la qualité des carcasses ?

Dans nos propres expériences comme dans d'autres, cette qualité apparaît inchangée chez les animaux traités et serait même, paríois, légèrement améliorée. Il en est de même du rendement (cf. Tableaux 2 et 3 ).

Comment se présente la viande des sujets traités?

Des divergences existent à ce propos. A notre avis, elles résultent des épreuves employées pour juger la qualité de cette viande.

D'une part, on utilise les dosages classiques de malières sèches, de protéines et de lipides; d'autre part, certaines méthodes de cuisson ménagée tendent à montrer qu'il n'y a pas de perte d'eau au cours de celles-ci. Nous sommes d'accord sur les premières épreuves; non sur les secondes. Elles n'ont, en effet, rien de commun avec ce qui se passe, en pratique, dans la cocotte familiale d'une ménagère ou dans le four de la cuisine d'un restaurant. Ici réside le malentendu.

Pour juger de la qualité de la viande MOERMAN a opéré de la façon suivante. Des échantillons, aussi identiques que possible, provenant du muscle psoas de 58 veaux ont été débarrassés de leurs graisses et de leur conjonctif, pesés, emballés sous vide, conservés 3 heures à $0^{\circ} \mathrm{C}$ puis chauffés une heure à $80^{\circ} \mathrm{C}$. Les paquets, une fois refroidis, sont ouverts. Le bouillon est rejeté et le morceau de viande pesé à nouveau. Le pourcentage de perte de poids est déterminé, renseignant ainsi sur la capacité de chaque catégorie de viande à lier l'eau. Le Tableau 5 rassemble les résultats obtenus.

\section{5}

Capacité à lier l'eau de viandes de veaux traités avec divers anabolisants

\begin{tabular}{|c|c|}
\hline Traitement & $\begin{array}{l}\text { Perte de poids (p.100) } \\
\text { après } 1 \text { heure de chauffage à } 80^{\circ} \mathrm{C}\end{array}$ \\
\hline A - témoir & $30,2 \pm 3,1$ \\
\hline g - $\quad 20 \mathrm{mg}$ Destradiol & $30,7 \pm 2,3$ \\
\hline 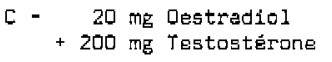 & $29,5 \pm 2,2$ \\
\hline $\begin{array}{l}0-\quad 20 \mathrm{mg} \text { Cestradiol } \\
+\quad 140 \mathrm{mg} \text { Trienbolone }\end{array}$ & $30,5 \pm 2,1$ \\
\hline 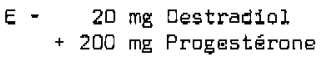 & $30,3 \pm 2,7$ \\
\hline
\end{tabular}
lots.

Il n'existe pas de différence significative entre les

Au cours des dosages que nous avons effectués sur les viandes des veaux soumis aux essais dont les résultats sont rassemblés dans le tablau 2, nous n'avons également pas trouvé de différences entre les lots. Les échantillons examinés, au nombre de 6 par lots, étaient constitués par les piliers du dia- phragme. On détermina, sur chacun d'eux, l'humidité sur le produit frais avant et après élimination des lipides, le taux des matières protéiques en l'état, enfin, on pratiqua, sur $10 \mathrm{~g} \mathrm{du}$ produit délipidé, le test de coction, c'est-à-dire la mesure de la quantité d'eau, exprimée en ml, éliminée par ces dix grammes au cours d'une cuisson au bain-marie. Les résultats obtenus sont présentés dans le tableau 6. 
Teneur en eau, en protéines, exprimée en p. 100 et " test » de coction de viandes de veaux ayant reçu différents implants

\begin{tabular}{|c|c|c|c|c|}
\hline $\begin{array}{l}\text { Sexe des veaux } \\
\text { et implants reçus }\end{array}$ & $\begin{array}{c}\text { Humidizé } p .100 \\
\text { sur vianda } \\
\text { fraiche } \\
\text { et fearts }\end{array}$ & $\begin{array}{l}\text { Humidité p. } 100 \\
\text { gur produit } \\
\text { dégralssé }\end{array}$ & $\begin{array}{c}\text { Matieres } \\
\text { protéidues } \\
\text { p. } 700 \\
\text { viande fraiche } \\
\text { ot Ecarts }\end{array}$ & $\begin{array}{c}\text { Test de cootion } \\
\text { (mg/10 g] } \\
\text { et Esarts }\end{array}$ \\
\hline $\begin{array}{l}\text { Mãles : } \\
\text { Diéthylstilboestrol }\end{array}$ & $\begin{array}{c}60,22 \\
(50,75-64,70)\end{array}$ & 75.6 & $\begin{array}{c}18,71 \\
(16,33-20,65)\end{array}$ & $\left(2,52^{2.31}+3,37\right)$ \\
\hline $\begin{array}{l}\text { Mòles : } \\
\text { Destradiol - Progesterone }\end{array}$ & $\begin{array}{c}60,59 \\
{\left[53,24^{-62,66]}\right.}\end{array}$ & 77.7 & $\begin{array}{c}15,79 \\
{[15,24=17,78]}\end{array}$ & $\begin{array}{c}2,24 \\
{[0,89-\exists, 17]}\end{array}$ \\
\hline $\begin{array}{l}\text { Femellez: } \\
\text { Oestradiol - Testosterrone }\end{array}$ & {$\left[60,58^{64,42}-71,81\right]$} & 76,9 & $\begin{array}{c}18,25 \\
{[17,36-20,10]}\end{array}$ & $(2,14-3,32)$ \\
\hline $\begin{array}{l}\text { Màlas : } \\
\text { Témoins }\end{array}$ & {$\left[\begin{array}{c}59,41 \\
{\left[53,39^{-64,14]}\right.}\end{array}\right.$} & 76,6 & {$[15,28-19,26]$} & $\left(1,15^{2,11}-3,17\right)$ \\
\hline $\begin{array}{l}\text { Fẹmellass : } \\
\text { Témoing }\end{array}$ & {$\left[57,97^{65,86}-71,76\right]$} & 78.1 & {$\left[\begin{array}{c}18,11 \\
{[16,03-20,3: 1]}\end{array}\right.$} & $\left(2,43^{3,00}-3,40\right)$ \\
\hline
\end{tabular}

Il n'y a pas de différences entre les divers lots. Sur d'autres échantillons, toujours au nombre de 6 par lot et constitués par les piliers du diaphragme, la cuisson globale réalisée pendant une heure en cocotte couverte, sans aucun apport de graisses, en se plaçant dans les conditions de la pratique, fait apparaître les rendements totaux suivants en jus dégraissé.

$\begin{array}{ll}\text { Lot Estradiol-Progestérone } & >12 \text { p. } 100 \\ \text { Lot aut DES } & <0,4 \text { p. } 100 \\ \text { Lot Estradiol-Testostérone } & >13 \text { p. } 100 \\ \text { Lots Témoins } & \begin{array}{l}\text { seule la graisse est } \\ \text { retrouvé dans la } \\ \text { cocotte }\end{array}\end{array}$

En considérant ces résultats, nous estimons qu'une association DES-TESTOSTERONE, comme on la préconise dans certains pays, demeure très discutable sur le plan examiné ici, sans parler, bien entendu, de l'aspect santé publique.

L'examen de ces différents chiffres fait apparaître certaines contradictions entre les tests effectués au laboratoire, en particulier le test de coction, et les résultats obtenus dans les conditions de la cuisson domestique. Dans ce dernier cas, la perte de "jus" est très importante avec les viandes de veaux implantés au DES et au mélange Estradiol-Testostérone. Les observations sur la perte de jus au cours de la cuisson furent confirmées par un cuisinier, en particulier en ce qui concerme le DES, lors de la préparation des viandes destinées à des essais de dégustation. Pour ces essais, 11 personnes examinèrent, sans connaître l'origine des viandes, l'aspect général, l'odeur, la saveur et la succulence, de rôtis de veau chauds ou froids. Les notes moyennes suivantes furent attribuées :

\begin{tabular}{|c|c|c|}
\hline $\begin{array}{c}\text { Rôti froid veaux } \delta \\
\text { implantés DES } \ldots\end{array}$ & 5,78 & (écarts : 4,2-8) \\
\hline $\begin{array}{l}\text { Rôti chaud veaux o } \\
\text { implantés DES ... }\end{array}$ & 6,50 & (écarts: $6,2-8$ ) \\
\hline $\begin{array}{c}\text { Rôti froid veaux } \delta \\
\text { témoins } \ldots \ldots \ldots \ldots \ldots\end{array}$ & 5,99 & (ćcarts: $4,7-8$ \\
\hline
\end{tabular}

Rôti chaud veaux o* témoins ............ 5,29 (écarts : 4 -8,2)

Rôti froid veaux ? implantés Gst.-Test. ..... non examinés

Rôtí chaud veaux $?$ implantés Est.-Test. ..... 4,69 (écarts : 3,3-8)

Rôti froid veaux $\rho$ témoins $\ldots \ldots \ldots \ldots \ldots \ldots$ non examinés

Rôti chaud veaux $g$ témoins ............ 4,7 (écarts : $3-7$ )

Rôti froid veaux ơ implantés Est..Prog. .... 5,58 (écarts : 5 - 8 )

Rôti chaud veaux $\delta$ implantés Est.-Prog. .... 6,88 (écarts: 5 -9,5)

Ainsi, les viandes des animaux témoins n'arrivent pas en tête du classement. La meilleure note est attribuée au rôti chaud provenant de veaux mâles implantés avec le mélange Estradiol-Progestérone. Ce sont les femelles, traitées ou non, qui obtiennent les notes moyennes les plus basses $(4,69-4,70)$. Il existe donc un décalage certain entre les examens de laboratoire et l'opinion des dégustateurs, ce qui ne nous étonne pas.

A la fin d'une autre expérience, seules les viandes provenant de sujets mâles implantés avec le mélange Estradiol-Progestérone et de sujets témoins furent examinées au laboratoire et dégustées par un groupe composé, à une ou deux exceptions près, des mêmes personnes que celles ayant opéré précédemment. Les essais faits au laboratoire furent modifiés. En particulier, l'épreuve de coction fut effectuée en plaçant un morceau de viande dégraissée pesant environ $50 \mathrm{~g}$ dans de l'eau bouillante sous réfrigérant puis en mesurant après 30 minutes la perte de poids. Les résultats obtenus sont les suivants :

Viande témoin .......... 44,8 p. 100

Viande Estrad.-Progestér. .. 42 p. 100

Viande Estrad.-Progestér. .. 42,1 p. 100 et 45 p. 100

L'analyse des viandes fraîches et cuites donne les compositions suivantes exprimées en g p. 100. 


\begin{tabular}{|c|c|c|c|c|}
\hline & \multicolumn{2}{|c|}{ Vezux tömoins } & \multicolumn{2}{|c|}{$\begin{array}{c}\text { Veaux Implantés } \\
\text { Destradial-Progestérone }\end{array}$} \\
\hline & Viande frazahe & Víande cuite & Viande fraighe & Viande cuite \\
\hline Humiditü & 75,26 & 62,67 & 75,25 et 74,31 & 54,71 et 82,63 \\
\hline Matière seche & 24,74 & 37,33 & 24,76 et 25,69 & 35,29 et 37,37 \\
\hline Matières protéiques & 22.5 & 34,9 & 23,5 et 23,5 & 33,5 et 35,2 \\
\hline Graisses & 1.45 & 3,25 & 1,18 et 1,06 & 1,82 et 2,70 \\
\hline $\begin{array}{l}\text { Hydraxyproline } \\
\text { (g p. } 100)\end{array}$ & $0,2 a$ & & 0,25 et 0,25 & \\
\hline
\end{tabular}

Ces résultats ne font apparaître qu'une faible différence dans le taux des lipides en faveur des viandes provenant des veaux ayant reçu des implants d'Estradiol-Progestérone. Ceci est très net après cuisson. Le taux de collagène, traduit par celui d'hydroxyproline, est semblable dans les diverses catégories de viandes fraîches.

Lors de la cuisson des viandes dont nous venons de parler, le cuisinier déclara que celle Témoin, dont il ignorait l'origine, avait rendu un peu plus de jus que les autres.

Les notes moyennes obtenues au cours de la dégustation sont les suivantes:

Veaux témoins non implantés 7,06 (écarts: $6 \quad-8,5$ ) Veaux implantés Estr.Prog.. 6,17 (écarts : 4,5 - 7,6) Veaux implantés (Estr.-Prog. 6,70 (écarts: 5,25-8)

Il paraît difficile de tirer des conclusions nettes de cet ensemble d'examens.

Au-delà des constatations mettant en évidence que les dégustateurs notent individuellement avec une certaine constance dans la sévérité ou l'indulgence et que, dans l'ensemble, ils ne donnent pas toujours la préférence aux viandes provenant des animaux témoins, deux faits apparaissent

$1^{\circ}$ L'implantation, quelle que soit sa nature, ne change pas la teneur en protéines d'une viande crue ou cuite de veau. Les écarts enregistrés, faibles d'ailleurs, ne sont pas significatifs. Ils tiennent à des variations individuelles dues sans doute à la composition du morceau lui-même, à son taux de lipides en particulicr;

$2^{\circ}$ Les viandes provenant des veaux mâles implantés au DES "rendent de l'eau "à la cuisson. Celles des veaux femelles implantés avec Estradiol-Testostérone également. De nombreuses ménagères l'ont constaté sans savoir que les veaux étaient implantés. Elles réagissent en disant: "La viande de veau " fond " dans la cocotte. " Est-ce pourtant une règle générale? Inversement les viandes des animaux témoins gardent leur eau et ne libèrent que la graisse. I1 en est de même des viandes des sujets mâles implantés avec le mélange (Estradiol-Progestérone. Dans l'ensemble, et tout au moins en se basant sur les chiffres du second essai, ces viandes seraient légèrement moins grasses, ce qui est un avantage diététique, sinon gastronomique, qu'il convient tout de même de confirmer.

On peut également estimer que, du point de vue organoleptique, l'implantation avec les hormones naturelles a peu our pas d'influences.

Quant aux conséquences de l'implantation sur l'apport protéique, elles sont nulles. Le consommateur n'est pas frustré. L'étude de la teneur en acides aminés des régimes destinés au rat dans lesquels la viande des veaux diversement implantés était incorporée à raison de 20 p. 100 , après avoir été préalablement lyophilisée, le confirme.

Pourquoi observe-t-on une libération d'eau dans les échantillons provenant d'animaux ayant reçu les implants des substances aux propriétés anabolisantes les plus réputées ? Il est difficile de répondre à cette question mais on peut penser qu'une synthèse protéique accrue pourrait entraîner une fixation d'eau supérieure à celle qui l'accompagne habituellement. Dans les conditions de cuisson classique où la température peut dépasser $150-180^{\circ} \mathrm{C}$, il y a libération d'eau, sans que, pour autant, l'apport alimentaire de protéines soit changé. Il est évident que d'autres recherches doivent encore être entreprises sur ce sujet. Retenons cependant que ni l'odeur, ni la saveur des viandes ne sont modifiées. KISEL a également noté cette absence de modification sur les viandes de veaux implantés au DES.

Aucun travail du même genre ne semble avoir été entrepris sur les viandes de veaux traités par le Zéranol ou la Trienbolone. S'il existe des résultats, on peut toujours leur reprocher d'avoir été obtenus dans des conditions plus théoriques que pratiques et, par conséquent, de ne pas correspondre à la réalité culinaire quotidienne, la seule valable.

Les autres inconvénients concernent les influences que les viandes, provenant d'animaux implantés, peuvent avoir sur le consommateur. Nous étudierons tout d'abord le cas du DES. En effet, de tous les produits employés, il paraît être celui le plus sujet à critiques. Il peut ainsi servir d'étalon de référence. Nous examinerons ses diverses influences et comparerons les autres anabolisants avec lui.

Le pouvoir cancérogène du DES, par ailleurs employé dans la thérapeutique du cancer de la pros. tate chez l'Homme, a été signalé. Les cancers du vagin observés chez des filles âgées de 15 à 20 ans auraient pour cause le traitement de leurs mères par le DES au cours de leur grossesse. Il était, en effet, courant, il y a quelques années, de prescrire des ostrogènes non stéroïdiques de synthèse aux femmes enceintes menacćes d'avortement spontané. Plusieurs observations ont mis en évidence cette action cancérogène à très long terme du DES. Elles ont conduit à considérer, non seulement les influences de traitements thérapeutiques, mais encore le problème des résidus d'œstrogènes non stéroïdiques de synthèse contenus dans la viande des animaux traités avec ces substances (cf. SINHA et DAO - 1872 et $X$ - 1971).

D'autres actions peuvent s'exercer sur l'appareil génital des animaux soit directement, soit à la suite de la consommation de viandes d'animaux implantés au DES.

Directement, KROES et Coll. (1971), après bien d'autres, observent, sur des veaux recevant en injection intrapéritonéale ou "per os" du DES, de l'Hexoestrol, du Dicenestrol et du benzoate d'Estradiol à des doses variant de 20 à $160 \mathrm{mg} / \mathrm{sujet}$, des changements profonds des testicules, de l'épididyme, des vésicules séminales, des canaux déférents, de l'uretère et de la prostate. Il existe, dans tous les organes accessoires du système génital, de la fibrose et de l'hypertrophie des muscles lisses. Nous rappelons que les modifications de la prostate peuvent, éventuellement, servir à mettre en évidence les car- 
casses de veaux traités au DES et aux autres hormones. De leur côté, MOHAN, GREESH et SAXENA (1972) ont observé que 3,75 et $5 \mu \mathrm{g}$ de propionate de DES donnés à des rates, diminuent le taux de conception de 50 p. 100 à 100 p. 100 respectivement. Cependant l'importance des portées nées de femelles non traitées mais saillies par des rats traités au DES n'est pas affectée bien que l'Estrogène abaisse le pourcentage des spermatozoïdes vivants, collectés au niveau de l'épididyme.

D'autres résultats prouvent que le DES affecte la sphère génitale en agissant vraisemblablement par le relais hypophysaire. Il s'agit pourtant là d'actions directes. Nous reviendrons sur elles.

Dans le contexte d'une utilisation du DES en élevage, les influences pharmacodynamiques sur le consommateur des viandes provenant d'animaux traités doivent surtout être prises en considération. Un premier point, mis en relief par VOGT, WALDSCHMIDT et KARG (1970) doit être souligné. C'est l'écart entre la sensibilité des méthodes de dosage d'une part et la teneur en résidus de la viande qui est fonction de la distance du point d'implantation ou d'injection. KARG (1973) a d'ailleurs bien souligné les différentes réactivités des organismes humain et bovin, ainsi que la sensibilité des méthodes utilisées actuellement pour le dosage des cestrogènes, en particulier, du DES. Les méthodes biologiques s'avèrent les plus sensibles pour le DES comme pour d'autres produits.

Dans les risques encourus par le consommateur, par suite de ces actions indirectes, on mettra également l'accent sur les individus dont la fonction hépatique est plus ou moins altérée. KLAASSEN (1973) a, en effet, montré que le DES est 140 fois plus toxique chez les rats dont le canal cholédoque est ligaturé. Il en est de même après ablation d'une partie du foie ou injection de $\mathrm{CCl}_{4}$. Il est ćvident que, dans le premier cas, le cycle enterhépatique est rompu et, dans le second cas, les possibilités de glycuro-conjugaison diminuées, voire même supprimées. Le taux d'excré- tion biliaire de DES est de $1.2 \mu \mathrm{g} / \mathrm{mn} / \mathrm{kilo}$ vif, chez les sujets dont une partie di. foie a été supprimée, contre $2 \mu \mathrm{g} / \mathrm{mo} / \mathrm{kilo}$ vif, chez les témoins. Le véhicule dans lequel est administré le DES modifie également la toxicité du produit en changeant sans doute sa vitesse de résorption.

Nous avons personnellement étudié, en utilisant la méthodologie dite de la toxicité des relais, l'action des viandes et des foies lyophilisés provenant de veaux traités au DES, au mélange đEstradiol-Progestérone ou Estradiol-Testostérone sur des souris et des rats considérés comme des pseudo-consommateurs. Nos résultats, déjà en partie publiés en 1971 et en 1972, mettaient en évidence, qu'après un an de consommation cle viandes de veaux traités avec divers implants dans les conditions de la pratique, les viandes des animaux ayant recu du DES rendaient toute reproduction impossible chez la souris comme chez le rat après avoir notablement réduit leur croissance. Le foie des veaux traités au DES, sans action pour la première porlée chez le rat et la souris, provoque toute de même chez le rat une nette diminution de la fécondité lors de la seconde portée. Une seule femelle sur 12 a mis bas dans le lot recevant, dans sa ration, 6 p. 100 de foie des sujets traités au DES contre une moyenne de 6 stur 12 dans les autres lots consommant un même pourcentage de foie provenant de veaux, soit non traités soit implantés à l'CEstradiol-Progestérone ou à l'Éstradiol-Testostérone. En effet, le foie comme les viandes des sujets inuplantés avec ces deux mélanges d'hormones n'altćraient ni la fécondité, ni l'appareil génital des anjmaux pseudo-consommateurs. Nous n'avons non plus noté aucun accident de cancérogénèse chez ces animaux. Les tableaux 7 et 8 , extraits d'un travail en cours de publication, rósument l'ensemble des résultats obtenus chez le rat.

Nos observations furent en partie confirmées par POKROVSKII et coll. (1972) dans une publication à laquelle il a déjà été fait référence.

Tableau récapitulatif du devenir des rates des groupes $\mathbf{M}$ recevant de la viande de veaux implantés diversement ou non implantés (T) 6 mâles et 12 femelles par groupe

\begin{tabular}{|c|c|c|c|c|c|c|c|c|c|c|c|c|}
\hline \multirow{2}{*}{$N^{0}$ des groupes } & \multicolumn{2}{|c|}{$1 M$} & \multicolumn{2}{|c|}{$2 \mathrm{M}$} & \multicolumn{2}{|c|}{$3 \mathrm{M}$} & \multicolumn{2}{|c|}{$4 M(T) *$} & \multicolumn{2}{|c|}{$5 M(T)$} & \multicolumn{2}{|c|}{$\begin{array}{l}\text { Ténains } \\
\text { élevage }\end{array}$} \\
\hline & $\sigma^{7}$ & 古 & $\sigma^{x}$ & q & 8 & q & $\theta^{*}$ & 古 & $\theta^{*}$ & $\begin{array}{l}0 \\
+\end{array}$ & $\sigma^{*}$ & 古 \\
\hline $\begin{array}{l}\text { Morts ou sactifiés en cours } \\
\text { d'exṕ̧rience }\end{array}$ & E & 12 & 3 & 4 & 3 & 2 & 1 & 1 & 2 & 4 & 3 & 5 \\
\hline Sacrifiés à 24 mois & & & 3 & 8 & 3 & 10 & 5 & 11 & 4 & a & 3 & 7 \\
\hline Paids mayen (g) a! secrifice & & 191 & 389 & 310 & 282 & 279 & 491 & 260 & 423 & 258 & 363 & 232 \\
\hline Poids moyen du penis [g] & & & 0,440 & & $0,230^{\circ}$ & & 0,368 & & 0,450 & & 0,380 & \\
\hline Poids moyen des terticuies (g) & & & 1,430 & & 0,910 & & 1,625 & & 1,495 & & 1.118 & \\
\hline Paids mayen des avalres + utérus [g] & & & & 0,510 & & 0.590 & & 0,890 & & 0,780 & & 0,660 \\
\hline Paids moyen du foie [g] & & & 14,38 & 10,36 & 9,71 & 10,32 & 15,45 & 9,81 & 13,59 & 9,86 & 12,25 & 8,87 \\
\hline Polds mayen des reins [g] & & & 1,520 & 1,220 & 1,305 & 1,110 & 1.495 & 1,450 & 1.445 & 1,065 & 1,470 & 1.000 \\
\hline $\begin{array}{l}\text { Nombre de portées sur deux séries } \\
\text { de saillies }[12 \text { o } \times 2=24]\end{array}$ & $0+0$ & $0^{\circ 0}$ & - & 15 & - & 15 & - & 13 & - & 13 & - & 14 \\
\hline $\begin{array}{l}\text { Pourcentege de tumeurs dans les } \\
\text { deux sexes ono }\end{array}$ & \multicolumn{2}{|c|}{$\begin{array}{l}\text { Néant 吉 } \\
12 \text { mols }\end{array}$} & \multicolumn{2}{|c|}{$22, z$} & \multicolumn{2}{|c|}{16,6} & \multicolumn{2}{|c|}{22,2} & \multicolumn{2}{|c|}{33.3} & \multicolumn{2}{|c|}{22,2} \\
\hline Nombre de tumeurs malignes & & & 0 & 0 & D & a & Q & $\square$ & 1 & 1 & 1 & 0 \\
\hline $\begin{array}{l}\text { Anomalies histologiques organes } \\
\text { Énitaux, foie et reins }\end{array}$ & $+\cdots$ & $+\cdots$ & 0 & 0 & Q & a & $\square$ & $\square$ & 口 & $\square$ & 口 & a \\
\hline
\end{tabular}

111 ; veaux implantes DES. Ce groupe a été sacrifié à $12 \mathrm{mois}$

2 M : veaux implantés oestradiol-progestërone

3 M : veaux implantés oestradiol-testastérone; 4 M et 5 M : témoín

: sur deux sujets seulement

: femelles saililies à trajs reprises et toujaurg infécondes; mâles avea dégénérescence des testicules

chez les femelles; il s'agit d'adénames mammaires communs chez le rat wistar

temolns communs aux eroupes $M$ et $F$

: 1 groupe 4 M non compté 1 ci socrifié a 12 mois avec $1 \mathrm{M}$ d seulement

$\because \quad$ : sans tumeurs des organes génitaux 
Tableau récapitulatif du devenir des rats des groupes $F$ recevant du foie de veaux implantés diversement ou non implantés $(T)$ 6 mâles et 12 femelles par groupe

\begin{tabular}{|c|c|c|c|c|c|c|c|c|c|c|c|c|}
\hline \multirow{2}{*}{$N^{j}$ des groupes } & \multicolumn{2}{|c|}{$\eta F$} & \multicolumn{2}{|c|}{$2 F$} & \multicolumn{2}{|c|}{$3 F$} & \multicolumn{2}{|c|}{$4 F[T]$} & \multicolumn{2}{|c|}{$5 F[T]$} & \multicolumn{2}{|c|}{$\begin{array}{l}\text { Têmoins } \\
\text { slevage }\end{array}$} \\
\hline & $\sigma^{7}$ & + & $0^{\pi}$ & $\stackrel{+}{+}$ & $\sigma^{\pi}$ & $\stackrel{q}{+}$ & $E^{M}$ & $\varsubsetneqq$ & $\nabla^{7}$ & $\stackrel{q}{+}$ & $\sigma^{a}$ & $\stackrel{9}{+}$ \\
\hline $\begin{array}{l}\text { Morts ou sacrifíśs en couss } \\
\text { d'expérience }\end{array}$ & 2 & 2 & 2 & 3 & 3 & 1 & 2 & 5 & $f_{\mathrm{r}}$ & & 3 & 5 \\
\hline Secrifiés entre 20 et 24 e mois & 4 & 10 & 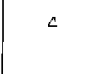 & $g$ & 3 & 11 & $=$ & 7 & 2 & 12 & 3 & $\begin{array}{c}7 \\
\text { nosis }\end{array}$ \\
\hline Poids mayen (g) au sacrifice & 381 & 257 & 350 & 242 & 372 & 285 & $37 \overline{0}$ & 305 & 444 & $23 c$ & 353 & 232 \\
\hline Paids mayen du penis (g) & $\begin{array}{l}\text { non } \\
\text { pesé }\end{array}$ & & 0,240 & & 0,410 & & 0,380 & & non & & 0,380 & \\
\hline Poids moyan des testicules [ $\mathrm{g}$ ] & 1,450 & & 1,462 & & 1,325 & & 1,355 & & $1,6.35$ & & $i .713$ & \\
\hline Poids moyen des ovalres + utérus [g] & & 0,748 & & 0.850 & & $\uparrow, 09 \square$ & & 0.890 & & 0,875 & & 0.550 \\
\hline Polds moyen du foie (g) & 13.69 & 5.99 & 13.01 & 10,09 & 16,68 & 10,71 & 12,79 & 71.59 & 15,57 & 9,71 & 12,25 & 3,37 \\
\hline Paids moyen des reins (g) & 1,410 & 1,081 & 1,580 & $i, 010$ & 1,593 & 1,125 & 1,420 & & 1,520 & 1.225 & 1,476 & 1,030 \\
\hline $\begin{array}{l}\text { Nombre de portées sur deux séries } \\
\text { de sailies }(12 \text { q } \times 2=24) \\
\text { Pourcentage des tumeurs dang les } \\
\text { deux sexes au }\end{array}$ & & 10 & & 15 & 96. & 15 & & 17 & 5. & 13 & 1 & 14 \\
\hline Nambre de tumeurs malignes & $\square$ & 0 & 0 & 0 & 0 & $i$ & Q & $\square$ & 1 & 0 & 1 & 1 \\
\hline $\begin{array}{l}\text { Anomalies histologiques organes } \\
\text { génitaux, faie et reins }\end{array}$ & D & 口 & 0 & 0 & 0 & 0 & 0 & 口 & 0 & Q & $\square$ & 0 \\
\hline $\begin{array}{r}1 \mathrm{~F}: \text { veaux implantés au DES. Sa } \\
2 \mathrm{~F}: \text { veaux implantés \&̊ pestradi } \\
3 \mathrm{~F} \text { : veaux implantés a oestradi }\end{array}$ & esst & 5 & fies & $\begin{array}{l}2 c \mathrm{ma} \\
z^{4} \text { ing }\end{array}$ & TEma & $=4 F$ & et 5 & $m \perp f$ & g $\mathrm{Z}$ & & & \\
\hline
\end{tabular}

Par ailleurs, nous avons déterminé que, chez le rat, la fécondité exprimée par le nombre de jeunes sujets sevrés par mère fécondée s'abaisse régulièrement et selon une droite pour des taux d'incorporation de DES au régime de base témoins de $6,20,35,60$ et $120 \mu \mathrm{g}$ par kilo d'aliment. On peut ainsi prévoir, qu'entre 100 et $120 \mu \mathrm{g}$, la stérilité totale s'établit. Rapportée à l'Homme de 60 kilos cette dose représente un apport total quotidien de $0,48 \mathrm{mg}$ qu'on peut arrondir à $0,5 \mathrm{mg}$ de DES. On doit toutefois noter que des quantités de résidus supérieures à $30 \mu \mathrm{g} /$ kilo n'ont jamais été trouvées dans les viandes lyophilisées utilisées dans nos expériences lors des dosages effectués par chromatographie en couche mince. Malgré cela des modifications graves des tractus génitaux mâles et femelles aboutissant à la stérilité, comme des diminutions de croissance, ont pu être mises en évidence chez l'animal pseudo-consommateur de viandes de veaux implantés au DES. Ces viandes agiraient donc, indirectement, comme agissent directement des doses de 100 à $120 \mu \mathrm{g} / \mathrm{kilo}$ d'aliment.

BROWING et coll. avaient montré, en 1958, que 0,6. 1,1 et $2,2 \mu \mathrm{g}$ de $\mathrm{DES} / 100 \mathrm{~g}$ de poids vif, correspondant en fait à des adjonctions aux aliments d'environ 90 , 135 et $275 \mu \mathrm{g} / \mathrm{kilo}$, donnés simplement trois jours avant la saillie ou durant la gestation et la lactation, n'influençaient ni la fécondité, ni la gestation mais perturbaient la lactation à ex juger par le retard de croissance des jeunes rats. L'action s'exerçait sur la prise alimentaire des femelles qui diminuait, comme nous l'avons d'ailleurs toujours observé au cours de nos expériences.

Ces résultats sont à confronter avec certains faits avant de se poser quelques questions.

Le métabolisme du DES et son cycle entérohépatique paraissent connus. HUBER et coll. (1972), travaillant sur des moutons, trouvent que la moyenne de rétention du DES marqué au $14 \mathrm{C}$ au niveau du foie est de 76,1 p. $100.38,5$ p. 100 de la radio-activité demeurant dans l'organe s'y trouve sous forme de
DES libre et 65,5 p. 100 sous forme glycuroconjuguée. La perfusion de l'organe montre une libération continue de l'activité initiale retenue. La muqueuse du rumen et celle de l'intestin peuvent aussi former et transporter le glycuroconjugué qui subirait également une hydrolyse dans les tissus. Un implant de $12 \mathrm{mg}$ de DES libérerait chaque jour 56 à $74 \mu g$ du produit dont 39 à $52 \mu \mathrm{g}$ seraient répartis hors đu foie. Il fau drait donc, dans ces conditions, entre 214 et 162 jours pour que l'implant soit totalement utilisé. Ceci semble long mais tout dépend de la nature du véhicule employé dans la préparation de l'implant et du cycle entérohépatique. Selon ASCHBACHER (1972), la plus grande partie du DES marqué au $14 \mathrm{C}$ et donné « per os " se retrouve dans les fèces. Nous avons pu le constater nous-même avec du DES tritié * en collaboration avec BORIES et VALETTE. ASCHBACHER ne trouve pas de DES dans l'air expiré et les échantillons de divers tissus et organes prélevés sur les trois moutons, 7 jours après l'ingestion du DES, ne renferment pas de teneurs mesurables en $14 \mathrm{C}$ à l'exception des surrénales.

A notre avis, il doit exister des différences entre le comportement du DES donné «per os » et celtui du DES administré en implant ou en injection. Ceci n'enlève rien à sa nocivité directe ou indirecte mais, à propos de cette nocivité indirecte, il faut souligner que selon GLASCOCK et SMITH (1970), travaillant sur des boucs et des brebis avec de l'Hexcestrol, la radio-activité liée aux protéines et non extractible serait élevée. Pour ces auteurs, les œstrogènes subiraient des transformations plus importantes chez les femelles et chez les ruminants. Ceci pourrait expliquer les influences néfastes de la viande de veaux implantés au DES.

Comment ces influences s'exerceraient-elles?

Dès 1971, CHANG MIN CHUEH et coll. montrent que, si l'implantation à la lapine d'Ethynylcestradiol (9,5 ou $2 \mathrm{mg})$ ou de DES $(0,5 \mathrm{mg})$, n'influence pas le

(") Résultats non publiés. 
développement de l'embryon, $2 \mathrm{mg}$ de DES empechent ce développement. Des rats mâles recevant à l'âge de 1 jour $1 \mathrm{mg}$ de DES montrent, trois mois plus taxd, selon MIETKIEWSKI et coll. (1970), des modifications morphologiques des neurocytes des noyaux de l'hypothalamus ainsi qu'tune augmentation des cellules du lobe antérieur de l'hypophyse. Les rats traités à la Testostérone, 3 mois après avoir reçu le DES, aveo des doses do $3 \mathrm{mg} /$ jour pendant 2 mois $1 / 2$, montrent des aspects histologiques et des réactions histochimiques normaux à la fin de ce traitement. On a d'ailleurs pu montrer, qu'à la suite du traitement de rats mâles avec le DES, le taux de Testostérone plasmatique s'abaisse et celui de prolactine s'élève. Il en est de même avec le meso-dihydrobutylstilbœestrol ou le $17 \beta$ Estradiol. Le DL. dihydrobutylstilboestrol demeure sans action. Ces observations de DANUTRA et coll. (1973) confirment, d'une certaine manière, celles de MAYER (1972) qui, chez le rat, note, sous l'influence du DES, une involution des testicules fixés à l'intérieur du péritoine. La Testostérone répare les dommages dus à l'cestrogène sans pour autant modifier ceux déterninés par l'action de la chaleur intraabdominale. Le Stilbostrol induit également l'ijvolution des testicules fixés sur la peau abdominale ou celle du scrotum.

Ainsi, directement, à des doses qui ne sont pas considérables, ou indirectement, le DES présente une série d'inconvénients que n'ont pas, indirectement, en toxicité de relais, les mélanges d'hormones naturelles Estradiol-Progestérone et Gstradiol-Testostérone. Dans le cas de ces mélanges il n'y a pas de résidus ou leurs taux sont insignifiants dans les viandes de veaux implantés 50 jours avant l'abattage (FERRANDO et coll. 1973). Il existe cependant des écarts dans l'estimation de ces résidus selon les différentes méthodes employées. Il apparaît également que peu d'implants sont retrouvés au moment de l'abattage. Nous n'en avons, en effet, découvert que sur 4 veaux parmi les 39 traités 50,60 ou 70 jours avant l'abattagc. Deux de ces implants récupérés ne renfermaient d'ailleurs plus trace d'hormone. En ce qui concerne la Trienbolone et le Zéranol (DAVID et coll. 1973), les résidus sont faibles sinon inexistants mais ces résidus ont ćté évalués par des méthodes chimiques et/ou biologiques sans que jamais n'ait été entreprise une étude de la toxicité de relais.

Dans le cas du Zéranol, on observe sur les animaux implantés des modifications, notamment de la prostate, comparables à celles observées chez les sujets traités avec des substances cestrogènes, c'est la raison pour laquelle nous estinnons que des études do toxicité de relais seraient souhaitables.

Ces divers faits relatés, on doit admettre, qu'étant donné l'existence de certaines contradictions et obscurités au sujet du DES, il a été sage de supprimer son usage. On peut d'ailleurs se poser un certain nombre de questions sur le retard mis à le faire quand on constate ses actions directes, même à doses relativement faibles, sur l'animal de laboratoire. Pourquoi son emploi a-t-il été maintenu si longtemps et sa suppression décidée, dans certains pays, en s'appuyant sur une action cancérogène observée chez la femme plutôt que ses effets sur la sphère génitale? Bien des produits, présentant directement sur l'animal de laboratoire de moindres influences, n'ont jamais été autorisés. L'examen de la toxicité de relais n'a fait que confimer les observations directement effectuées tout en soulevant une série de problèmes, pour l'instant difficiles à interpréter et délicats à résoudre, même en utilisant les méthodos les plus sensibles.

Nous avons également toujours pensé que la clause DELANNEY, interprétée de façon trop étroite, obnu- bile certains esprits. Ils ne songent qu'au seul cancer et négligent des faits d'apparence plus banale mais ayant des conséquences aussi graves sur d'autres plans.

Quelles positions adopter pour utiliser les anabolisamts dans la pratique?

Nous estimons, quant à nous, nécessaire de ne les utiliser que sous contrôle vétérinaire. Telle était déjà, en 1967, l'opinion de KARG. WILLEMART (1973) a bien étudié la qucstion. Nous nous rangeons à ses conclusions tout en estimant que TOUS les anabolisants devraient d'abord être soigneusement expérimentés mais, même une fois admis à être utilisés, il serait souhaitable de les inscrire au tableau. Aucun d'entre eux ne devrait, pour l'instant, être utilisé librement. Telle sont, à notre avjs, les règles dont il convient que les pouvoirs publics s'inspirent en tous pays et spécialement dans des pays comme ceux du Continent Africain où de mauvaises habitudes n'ont pas encore été prises.

\section{III. - CONCLUSIONS GENERALES}

L'élevage s'industrialise en Europe et en Amérique du Nord. Cette industrialisation implique l'apparition de nouvelles structures et l'utilisation de nouvelles méthodes. Tout ceci nécessite des organisations complexes et, en particulier, un changement dans l'inspection des produits d'origine animale qui en même temps que morphologique et bactériologique devra être de plus en plus chimique. Ici réside déjà une première difficulté.

Il en est une autre pour les pays d'Afrique.

Qu'ils se gardent de tomber dans le mythe du pro. duit miracle et de l'additif susceptible de pallier toutes les carences. Rien ne sert d'employer de telles substances quand un minimum de couverture des besoins et de respect de l'équilibre alimentaire n'est pas assuré. C'est le premier but auquel il convient de parvenir mais il faut l'atteindre sans pousser trop loin le perfectionzisme. La tentation de beaucoup de chercheurs et d'éleveurs d'Europe et d'Amérique fut, et demeure, la recherche de cetle perfection. La productivité, avons-nous dit avec SCHALLER, n'est pas la rentabilité.

Avec des rations présentant des déséquilibres non excessifs, notamment au niveau de la couverture du besoin protéique, les additifs et, en ce qui nous concerne ici, les anabolisants, peuvent permettre d'obtenir de meilleurs résultats tout en économisant des quantités non négligeables de protéines. Encore faut-il assurer un minimum de couverture des autres besoins. Au niveau de la perfection, la marge de gain obtenue grâce à ces diverses substances, dont les anabolisants font partie, demeure incertaine. Le coût du produit s'ajoute à celui de la recherche d'un parfait équilibre alimentaire. On s'efforcera de juger la situation avec bon sens. On recherchera peut-être beaucoup plus un équilibre économique qu'un équilibre alimentaire. L'anabolisant sera utilisé pour atteindre cet équilibre économique malgré une certaine relativité de l'équilibre alimentaire. Cependant on ne devra pas employer n'importe quel anabolisant. Ceux-ci doivent, tout en étant efficaces, ne compromettre ni la qualité des viandes, ni la santé des consommateurs. Quelques-uns d'entre eux semblent remplir ces conditions mais seuls les vétérinaires devront les utiliser.

Il convient, en effet, dès à présent, de songer à cette qualité et à la protection de cette santé. Daris ces pays d'Afrique, célèbres par leurs sorciers, on ne 
substituera pas, sous un prétexte de modernisme, de nouvelles pratiques critiquables à d'autres qui paraissent parfois curieuses quand on les juge superficiellement.

La législation des Etats africains doit dès maintenant tenir compte de ces nouvelles techniques. Les structures de ces Etats permettront, sans doute, de résoudre certains problèmes plus facilement que nous pouvons le faire.

Le bon sens est une qualité essentielle des éleveurs. Qu'ils ne l'oublient pas. En zootechnie, les voies d'apparence plus faciles se révèlent souvent pleines d'embûches, de difficultés et, finalement. de đébuires.

\section{BIBLIOGRAPHIE}

1. ASCHBACHER (P.W.), J. Anim. Sci., 1972, 35, 1031.

2. BAKER (F.-H.), ARTHAUD (V-H.), J. Anim. Sci, 1972, 35, 752 .

3. BEESON (W.-M.), ANDREWS (M.-S.), PERRY (T.-W.) J. Anim. Sci., 1956, 15, 679.

4. BOGDANOFF (M), KASNEDELTCHEFF (M.), Rec. Méd. Vét., 1972, 148, 577 .

5. BORGER (M.-L.), WILSON (L.-L), SINK (J.D.), ZIE GLER (J.-H.), DAVIS (J.-L.), J. Anim. Sci., 1973, 36, 706

6. BRADLEY (N.-W.), BOLING (J.-A.), LUDWICK (R.-L.) J. Anim. Sci, 1972, 34, 497.

7. BROWNING (C.-B), PARRISH (D.-B.), FOUNTAINE (F.-C.), J. Nut., 1958, 66, 310 .

8. BRUEGGEMANN (J.), TIEWS (J.), GROPP (J.) BOEHNCKE (E.), Wien. Tierarzt1, Monatsschr., 1973 60, 92 .

9. BURROUGHS (W.), CULBERTSON (E.), CHENG (W. H.) - HALE, HOMEXER P., I. Anim. Sct., 1955, 14, 1015.

10. BUTENANDT (A.), JACOBI (H.), Hoppe Seyler Z., 1933 218, 104 .

11. CANTINI (G.), CERUTISCURTI (J.), FIUSSELLO (N.), Folia Vet. Latina, 1973, 3, 203.

12. CEDARD (Lise), Les ostrogènes naturels. Biosynthèse ct métabolisme. 1 vol. Masson édit. Paris, 1965. 203 pages.

13. CHANG MIN CHUEH, CASAS (J.-H.), HUNT (D.-M.), Fert. Sterit., 1971, 22, 388.

14. DANUTRA (V.), HARPER (M.-E.), BOYNS (A.-R.), COLE (E.-N.), BROWNSEY (B.-G.), GRIFFITHS (K.), J, Endocrinol, 1973, 57, 207.

15. DAVID (C.), GRANDADAM (A.), PUPIN (F.), SZUMOWSKI (P.), THERET (M.). VÁISSAIRE (Y.)' VUIL LAUME (R.), WYERS (M.), Rec. Méd. Vét., 1973, 149, 503.

16. DAVIS (J.-L.), GARRIGUS (V.-S.), J. Anim. Sci.. 1971 32, 107 .

17. FERRANDO (R), Les bases de l'alimentation; Vigot Frères, Edit. (2* édit.) 1964.1 vol. 388 pages.

18. FERRANDO (R.), C.R. Acud. Sci., (Série D), 1971, 273, 1224 .

19. FERRANDO (R.), Procced. international meeting on use of oestrogens in cattle Breeding. Alfort Ecole Nale Vét. Lab. Nut. Alim. ; publ. 1973, 79 pages.

20. FERRANDO (R.), BOIVIN (R.), C.R Acad. Sci., (Série D), 1972, 274, 251.

21. FERRANDO (R.), TRUHAUT (R.), C.R. Acad. Sci., (Sćrie D), 1972, 275, 279 .

22. FERRANDO (R.), GUILLEUX (M.M.), GUERILLOTVINET (A.)., Nature (London), 1961, 192, 1205.

24. FERRANDO (R.), MIlc HENRY (N.), VALETTE (J.P.), PARODI (H.), C.R. Acad. Sci., (Série D), 1972, 274, 2240.

25. FERRANDO (R.), VALETTE (J.-P.), BOIVIN (R.), PARODI (H.), sous presse (1973) dans C.R. Acad. Sci., (Série D).

26. GAVRISHCHUK (V.-I.), KALININA (K.-N.), d'après Chemical Abstr., 1973, 78 143904

27. GLASCOCK (R.-F.), SMTTH (R.-W.), Ann. Biol anim. Bioch. Biophys., 1970, 10, 343.

28. GLEY (P.), MENTZER (Ch.), C.R. Soc. Biol., 1945, 139, 1055

29. GOODRICH (R.-D.), MEISNE (J.-C.), KOLARI (O.-E.), HARVEY (A.-L.), AUNAN (W.-J.), HANSON (L.E.), Stil- boestrol studies with beef cattle. Univ. Minnesota. Agr. Exp. Station, 1967, Bull $\mathrm{n}^{\circ} 486$

30. GRANDADAM (J-A.), SHEID (I.P.), DREUX (H.), Rec. Med. Vét., 1973, 149, 793 .

31. GRANDADAM (J.A.), SHEID (J.-P.), DREUX (H.), BRUERRE (D.), Rec. Méd. Vét., 1972, 148, 1137.

32. GREBING (S.-E.), HUTCHESON (D.-P.), PRESTON (R. L.), J. Anim. Sci., 1970, 31, 763.

33. HUBER (I.-L.), Can, J. Physiol. parmacol, 1970, 48, 573

34. HUBER (T.-L.), HORN (G.-W.), BEADLE (R.-E.), J Anim. Sci., 1972, 34, 786

35. KARG (H.), Tierärz. Wochenschrift, 1962, 75, 124.

36. KARG (H.), Economie Méd. animt., 1962, 5, 337.

37. KARG (H.), Der Tierziichter, 1964, 21, 121.

38. KARG (H.), Archiv. Lebenomittelhyg., 1964, 6 et 7, 1 .

39. KARG (H.), Symposiuxu international, Salzburg, 1967.

40. KARG (H.), Oestrogens and public Hygiene: The problems of control by special considerations of own experiments. - Proceed. International meeting on use of oestrogens in cattle breeding. Alfort Ecole Nale Vél. Lab. Nut. Alim. publ., 1973, pp. 37-47.

41. KISEL (I.-V.), Sb. Rab. Leningrad Vet. Inst., 1971, $\pi^{\circ} 32$, 224.

42. KLAASSEN (C.-D.), Toxicol. Appl. Pharmacol, 1973, 24, 142 .

43. KROES (R.), BERKVENS (J.-M.), LOENDERSLOOT (H.-J.), RUITENBERG (E.-J.), Zentralbl. Veterinaermed. Reihe A., 1971, 18, 717

44. LAZAR (G.), Endocrinol., 1973, 61, 152.

45. LOOSE (L.D.), BREITENBACH (R.P.), BARRETT (J T.), Compar. Biochem. Physiol., 1973, 45, 587.

46. MATSUSHIMA (A.), CLAUTON (R.), 5' Congrès Intern. Nut., Washington, 1960, Res. 121

47. MAYER (G.), Ann. Endocrinol., 1972, 33, 107.

48. MIETKIEWSKI (K.), MISKOWIAK (B.), LIMANOWSKI (A.) Osrodokova Koutr. Przyasdki. Mater. Kouf., 1970 24-26 dans Chem. Abstr., 1972, 76, 149.313.

49. MIROCHA (C.-J.), CHRISTENSEN (C.-M.), NELSON (G.-H.), Appl. Microbiol., 1969, 17, 482.

50. MOERMAN (P.-C.), Water binding capacity of the musculus psoas minor from calves traited with anabolics. Proceed. International meeting on use of oestrogens in cattle breeding. Alfort Ecole Nale Vét. Labo Nut. Alim. publ., 1973, D. 30 .

51, MOHAN GREESH, SAXENA (S.K.), Res. J., 1972, 6, 95.

52. Nal. Acad. Sci. - Nal. Res. Council. - Hormonal relationships and applications in the production of meats, milk and eggs, 1966, Washington D.C., Publ, $\pi^{\circ} 1415$.

53. PADUCHiEVA (L.-L.), Zhivotnovodstvo, 1972, 2, 20.

54. POKROVSKII (A.A..) NESTERIN (M.F.) VAVILINA (G.-P.), RYAZANTSEVA (E.-E.), SOLOV'EVA (L.-Ya.), Vesin. Akad. Nauk. SSSR, 1972, 27, 3 .

55. POTTIE (G.), Wlaams Diergen. Tieljschrift., 1972, 132, 401

57. RALSTON (A.-T.), TAXLOR (N.-O.), DAVIDSON (T. P.), Effect of DES on growth and carcass quality of beef cattle, Oregon Agr. Exp. Station, 1969, Bull. Techn. $n^{\circ} 110$.

58. SHARAF (A), GOMAA (N.), Qual. Plant. Mater Veg., 1971, 20, 279.

59. SINHA (D.), DAO (Th.), Estrogen target tissues neoplasia, Proc. Workshop, 1970 (Publ. 1972), 307-316. 
60. STANDARA (S.), CHURY (J.), Acta Vet. (Brno), 1973, 41, 251 .

61. TIEWS (J.), GROPP (J.), BOEHNCKE (E.), Studies on the effect of an anabolic agent (oestradiolbenzoateTrienbolone) on N-retention in fattening calves, in Proceed internal. meeting on usc of oestrogens in cattle brecding. Alfort Ecole Nale vét. Lab. Nut. Alim. publ., 1973, 7-11.

62. VOGT (K.), WALDSCHMIDT (M.), KARG (H.), Berl. Mïrichn. Wschr, 1970, 83, 457.

63. VAN DER WAL (P.), BERENDE (P.-L.-M.), note à 65th An. Meeting Amer. Soc. Anim Sci., Lincoln, Nebraska, 31 july 1973.

64. VAN DER WAL (P.), Van WEERDEN (E.-T.), note à 65th Ar1. Meeting Amer. Soc. Anim. Sci., Lincoln. Nebraska, 31 july 1973.

65. VAN DER WAL (P.), Van WEERDEN (E.J.), BERENDE (P.-L.-M.), Van HELLEMOND (K.-K.), The influence of anabolic on performance and N-retention of veal calves. in Proceed Internal. meeting on use of oestrogens in cattle breeding. Alfort Ecole vêt. Lab. Nut. Alim. publ., $1973,13-25$.

66. WARR (G.-W.) SLJIVIC (V.-S.), Brit. J. Exp. pathol., $1973,54,56-68$ et $69-78$

67. WILlemaRT (J.-P.), Bull. Soc. vét. Pratique, 1973, 57, 267.

68. WILLIAMS (R.T.), The fate of phenolic compounds in the body, dans The pharmacology of plant phenolics Academic Press., London, 1959, p. 13.

69. WILLWOCK (J.), Tieraerztl. Umsch., 1972, 27, 343-344 et 348.

70. X... Le cancer qui venait des oestrogènes, Méd. Mondiale, $1971, \mathrm{n}^{\circ}$ 90-117.

71. $\mathrm{X}$...., Estrogènes chez la mère, cancer chez l'enfant, Instant. méd., 1972, 23, 226.

(Cf. à ce sujet HERBST (A.-L.), KURMAN (R.-J.), SCULLY (R.E) POSKANZER (D.G.), New Eng. J. Med., 1972, 287, 1259 el HUSS (K.-S.), I.A.M.A., 1971, 218, 1564. 